\title{
Analyse des risques et dispositif de suivi : Le cas d'Izaute et Lussagnet
}

\author{
par Yves Lebas et Lionnel Thomas \\ d'Elf Aquitaine Production
}

La pertinence technique et économique des stockages de Lussagnet, depuis 1957, et Izaute, depuis 1983, dans le SudOuest de la France n'a jamais été contestée. Les caractéristiques géologiques des anticlinaux dans des sables aux qualités de perméabilité et porosité particulièrement convenables et la bonne insertion dans le système de productiontransport de gaz naturel les justifient amplement.

Aux premières préoccupations concernant la sécurité des installations de surface vis-à-vis de l'environnement proche s'est progressivement substituée une interrogation spécifique stocker du gaz naturel dans un aquifère à eau potable ne risque-t-il pas de détériorer ou appauvrir une ressource vitale ?

Le seul fait que la question se pose incite certains à considérer que de tels stockages sont inenvisageables. Au travers de sa pratique, d'une confrontation suivie avec les experts et les scientifiques, de la nécessité d'expliquer et justifier auprès des Pouvoirs Publics ou directement auprès des populations concernées, Elf a été conduit à proposer des réponses de plus en plus précises à cette question légitime.

Sans doute le débat de principe n'est-il pas clos. Mais une appréciation plus exacte de la réalité des risques encourus, et donc des moyens d'y faire face ou mieux encore de les prévenir, nous conduit à affirmer que l'on peut concilier les besoins croissants de modulation saisonnière des usagers du gaz et la préservation de la qualité, comme des quantités disponibles, de cette ressource qui pourrait devenir rare qu'est l'eau.
Cette communication a pour but, en présentant l'ensemble du dispositif de suivi (I) illustré au travers de l'exemple particulier du suivi physico-chimique des eaux (II) de montrer comment le travail d'interrogation, confrontation, explication opère en tant que réducteur d'incertitude. Mais il ne suffit pas de réaliser des travaux de prévention des risques encore fautil les faire connaître; les dispositifs d'information et contrôle (III) progressivement mis en place sur Izaute et Lussagnet à l'occasion des autorisations d'exploitation accordées dans le cadre des règlements existants, ont aussi cet objet.

\section{I 圈 UN DISPOSITIF DE SUIVI QUANTITA- TIF ET QUALITATIF}

Les risques vis-à-vis de la nappe peuvent relever soit de phénomènes mécaniques, les variations de pression engendrées par les stockages et leur diffusion dans l'aquifère (1.2) soit de phénomènes physico-chimiques, les modifications que le gaz et ses composants pourraient entraîner sur la qualité de l'eau (1.2). Pour cela un réseau de surveillance du stockage (gaz et aquifère) a été mis en place (1.1).

\subsection{Le réseau de suivi}

Au-delà du strict périmètre technique des stockages qui regroupe les puits de suivi de la bulle de gaz et de qualité de

The natural gas storage in the drinkable aquifer of Lussagnet sands has raised a specific question: isn't there any risk to deteriorate this vital resource or to nake it poorer ?

In order to answer to this question, Elf, in accordance with the public authorines, has carnied out studies and researchs aiming to assess the risks incurred, and implemented a permanent follow-up device to prevent them.

This aricle which is illustrated by a follow-up of the physicochemical quality of waters aims to show how a new approach, conciliating the gas storage and the preservation of the aquifer, could be developed. 


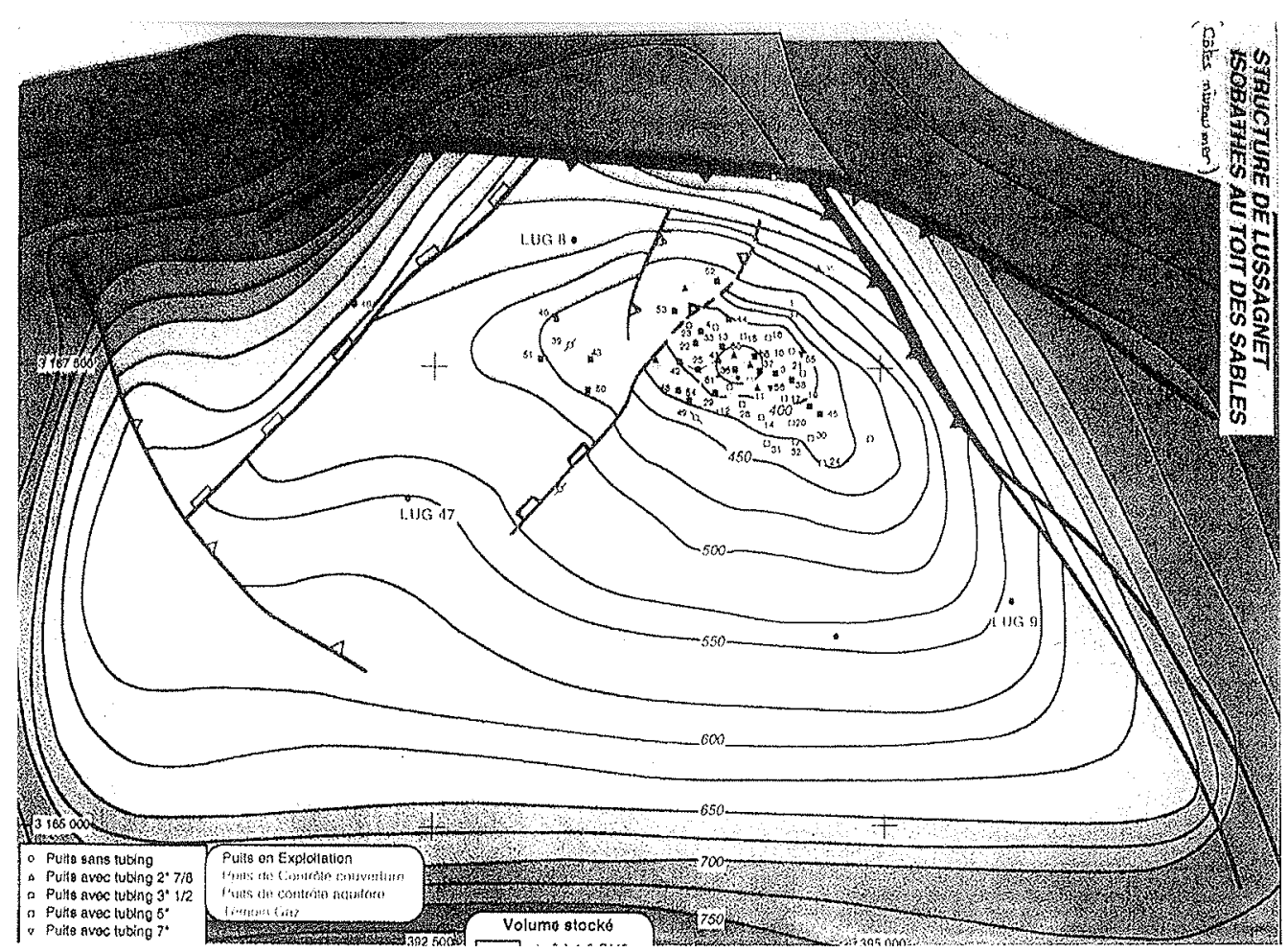

\section{RESEAU DE SURVEILLANCE DES NAPPES}

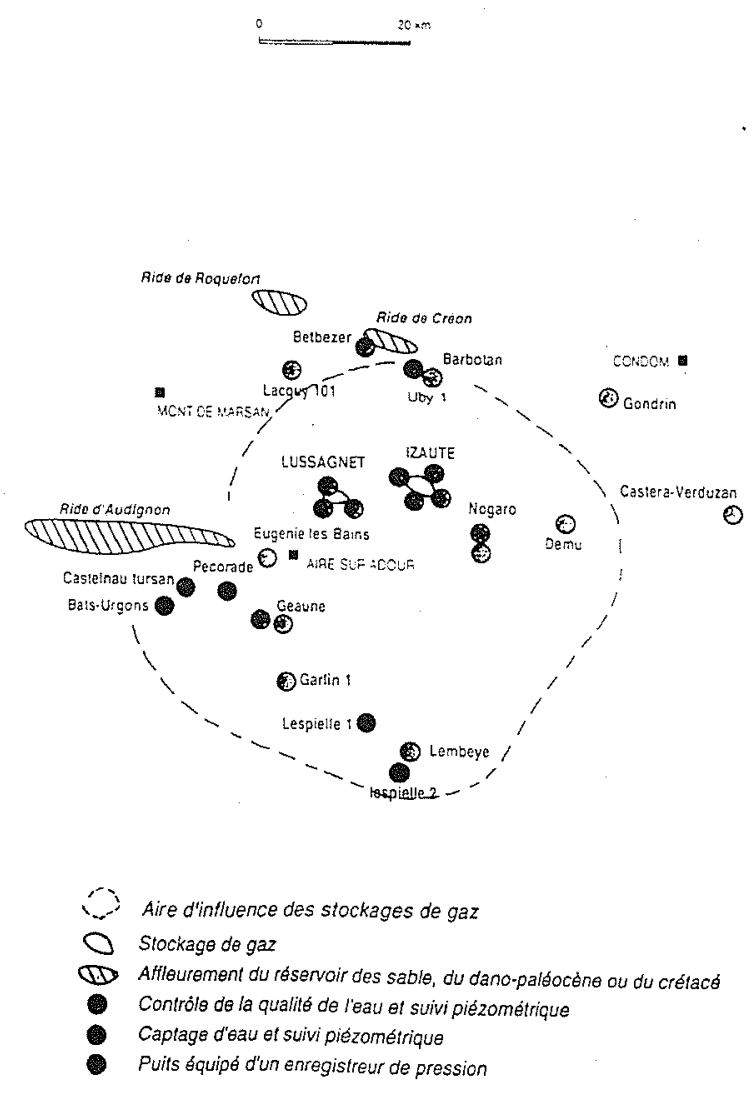

l'eau ainsi que les puits d'exploitation (cf. planche l), le réseau de surveillance s'étend sur un rayon de $50 \mathrm{~km}$ autour d'Izaute et Lussagnet.

Ce réseau recouvre la zone d'influence des stockages proprement dite, zone définie par les relevés de pression où l'on constate des battements supérieurs à $\pm 5 \mathrm{~m}$ en phase avec la saisonnalité des stockages. Cette zone est comprise entre 20 et $40 \mathrm{~km}$ autour des stockages (cf. planche 2).

Le dispositif de surveillance comporte aujourd'hui 45 puits, dont 35 pour le suivi de l'aquifère. Parmi ceux-ci, qui enregistrent tous les variations piézométriques, 13 sont plus particulièrement utilisés pour les analyses qualitatives régulières, 9 dans le périmètre de stockage, 4 dans la zone d'influence. Plus spécifiquement 1 puits (IZA 101) est perforé dans J'aquifere sous-jacent du Dano-Paléocène, au titre du contrôle de celui-ci.

Ce réseau est évolutif : il doit intégrer les nouveaux puits forés dans la zone d'influence et substituer tel ou tel puits dont la fonction peut changer à l'intérieur du périmètre. Au cours des années ce réseau s'est resserré, avec le souci d'assurer la continuité des séries sur les mesures effectuées. Aux puits de suivi et contrôle de l'aquifère il faut ajouter les puits de contrôle de l'imperméabilité de la roche couverture des stockages, du suivi de pression de la bulle de gaz et de témoin de l'interface gaz-eau (1 à 3 par stockage).

\subsection{Le suivi piézométrique}

La création de deux stockages dans les Sables de Lussagnet a superposé à la baisse piézométrique entraînée par une exploitation croissante de l'aquifère pour les besoins agricoles et domestiques les variations de pression engendrées par les mouvements saisonniers des stockages.

Le réseau de piézomètres, chargé de contrôler la piézométrie des sables permet d'une part de prévenir les effets des variations de pression pour les autres utilisateurs, d'autre part de délimiter la zone d'influence des stockages. 
En termes d'environnement, ces variations peuvent entraîne deux types de conséquences si, dans la zone d'influence, il existe des exutoires ou des communications avec des aquifères sous-jacents.

\section{- Les exutoires : observations et mesures}

Dans ce cas, il faut veiller à ce que la piézométrie à l'exutoire, ne dépasse pas une cote bien définie. Les Sables de Lussagnet affleurent à Barbotan : cet affleurement est d'ailleurs à l'origine d'une très ancienne activité thermale.

Lorsque la station thermale est fermée il apparaît un tropplein qui correspond à la surpression engendrée par les stockages. Cela représente environ $100000 \mathrm{~m}^{3}$ par an, soit $0,7 \%$ de la consommation annuelle de l'eau des Sables $\left(14 \mathrm{Mm}^{3}\right.$ en 1994).

\section{- Les communications entre aquifères}

Les communications entre aquifères activées par les variations de pression de la nappe provoquées par les phases d'exploitation des stockages supposent soit l'interruption de la barrière géologique entre aquifères soit l'existence de failles non étanches en son sein.

Avec l'appui des Universités de Bordeaux III et Paris VI et en partenariat avec les Pouvoirs Publics et les collectivités locales concernées, Elf a engagé une série de travaux pour vérifier l'existence ou non de telles communications : analyse sismique et géologique, jaugeages de rivières, exutoires du Dano-Paléocène (aquifères sous-jacents aux Sables) et suivi piézométrique de celui-ci au droit des stockages, recherche de fluctuations dans les concentrations d'éléments physico-chimiques des eaux selon la phase d'exploitation des stockages.

A ce jour aucune mesure n'est venue confirmer l'hypothèse de communications activées par les stockages et susceptibles de provoquer par mélange d'eaux de qualités différentes une altération des «bonnes eaux» par de «mauvaises eaux ».

\section{- Le modèle mathématique}

L'effet des deux stockages sur la nappe est mesuré à travers un modèle mathématique construit en 1987. Il figure le comportement en pression du réservoir des sables de Lussagnet et intègre le fonctionnement de l'ensemble de la région Aquitaine depuis son alimentation par les eaux infiltrées le long du piémont Pyrénéens ou des rivières du Périgord jusqu'aux consommations de la région bordelaise.

La prise en compte des données concemant la gestion de la ressource par les autres utilisateurs locaux (la station thermale de Barbotan ou les usages agricoles par exemple) permet de calculer précisément les variations de pression résultant de l'accroissement du niveau de stockage et de fixer un programme d'exploitation dons les effets sont mâ̂trisés.

Ce modèle de gestion constitue également un instrument de vérification des hypothèses géologiques et hydrogéologiques qui le sous-tendent: ses bons résultats opérationnels - conformité des calculs et des mesures - supposent qu'aucun phénomène majeur insoupçonné ne provoquera d'inversion de la logique.

\subsection{Le suivi qualitatif}

Prévenir une pollution de l'eau par le stockage de gaz nécessite de préciser la composition du gaz injecté, ce que les environnementalistes appellent le terme source, puis d'analyser les mécanismes qui interviennent dans le transport de masse pour en vérifier l'importance relative, de vérifier enfin l'absence d'altération des caractéristiques physico-chimiques et bactériologiques des eaux dans le pourtour des stockages.

\section{- Le gaz injecté}

Dans le cas du gaz, trois types d'éléments sont à suivre : les hydrocarbures combustibles, les métaux lourds et l'odorisant THT (Tétrahydrothiophène), auquel il faut ajouter le soufre et ses dérivés.

En ce qui concerne les deux premiers les études théoriques menées en commun avec le Laboratoire d'Hydrogéologie de l'Ecole des Mines de Paris et l'Institut Français du Pétrole ont montré le caractère très limité d'éventuelles diffusions, en deça des normes de pollution même à terme lointain.

Les mesures effectuées à ce jour n'ont décelé aucune diffusion de méthane dans la nappe (représentant $96 \%$ du gaz injecté, sa petite taille moléculaire en fait un traceur parfait) et n'ont pu déceler aucune présence de métaux rares.

Pour ce qui est du THT (odorisation du gaz) la recherche menée en commun avec Gaz de France et en liaison avec les Universités de Pau et Montpellier ainsi qu'avec le CNRS n'est pas encore achevée. Au-delà de la complexité des mécanismes étudiés de dissolution et d'adsorption par la roche du THT injecté on peut-être estimé que le phénomène est endigué dans la bulle de gaz et le considérer comme réversible.

\section{- La qualité de l'eau}

Le suivi qualitatif de l'eau mis en place en 1957 lors de la création de Lussagnet comporte un suivi micro-biologique et un suivi physico-chimique. Il vise à s'assurer que l'état initial est respecté et qu'aucune altération n'apparaît.

Le suivi microbiologique a pris en compte d'une part l'analyse chimique des nutriments bactériens d'autre part l'étude quantitative de la flore bactérienne. Le bilan établi en 1994 a montré que l'aquifère entourant le stockage demeure un milieu très pauvre cumulant les limitations nutritionnelles des bactéries et empêchant dont tout développement microbien notable. Aussi seules des analyses de veille ou d'alerte sont maintenues (dénombrement de la flore totale par épifluorescence et dosages de $\mathrm{H} 2 \mathrm{~S}, \mathrm{SO} 4$ et S total)

Le suivi physico-chimique s'appuyant sur des relevés réguliers s'est d'abord préoccupé de repérer d'éventuelles anomalies par rapport aux valeurs initiales. A la demande du Comité de Suivi, Elf a développé depuis deux ans un outil statistique facilitant l'interprétation d'éventuelles évolutions même à l'intérieur des normes acceptées.

\section{LE SUIVI PHYSICO-CHIMIQUE DE LA QUALITÉ DES EAUX}

Les chroniques du suivi qualitatif mis en place depuis plus de 30 ans, et constamment complété depuis, permettent aujourd'hui, grâce à l'exploitation statistique, utilisée complémentairement à l'interprétation conventionnelle de résultats par le chimiste, d'apporter des précisions quant à l'incidence des stockages sur la qualité des eaux. 


\subsection{Le dispositif de suivi}

Le réseau de surveillance établi sous le contrôle des Pouvoirs Publics comporte 13 puits. Les relevés peuvent être annuels, semestriels pour vérifier d'éventuelles variations saisonnières liées au mouvement engendré par les stockages, voire mensuels quand une attention particulière est nécessaire sur une période limitée.

Les critères analysés sont les suivants :

- paramètres organoleptiques,

- éléments majeurs,

- degrés et titres,

- indices chimiques de pollution,

- balances ioniques,

- agressivité,

- métaux,

- éléments traces, anormaux et toxiques,

- analyse bactériologique.

La plupart de ces déterminations sont effectuées par différents laboratoires de la communauté scientifique publique et privée. Toutefois, l'analyse de cohérence tout d'abord, l'étude critique des résultats ensuite et enfin les traitements statistiques sont conduits par le laboratoire de l'usine de Lacq.

La réussite de cette collaboration repose sur un travail précautionneux, d'harmonisation des méthodes analytiques entre les différents intervenants. De plus, il est nécessaire de déterminer pour chaque technique des critères essentiels tel que répétabilité, reproductibilité, limites de détection, de quantification, indices de capabilité, etc.

\subsection{L'interprétation physico-chimique des données}

Il s'agit d'une analyse descriptive des résultats, eu égard aux diverses exigences réglementaires concernant la qualité des eaux.

La représentation des eaux sous la forme de diagrammes de Schoeller, est retenue dans le but de caractériser leurs provenances, leurs particularités, leurs similitudes (cf. planche 3). Des différences significatives pourraient traduire des phénomènes exogènes aux stockages (pollution, communication entre différents réservoirs...).

A l'heure actuelle, ce mode d'exploitation met en évidence de légères différences de qualité d'eaux prélevées dans les puits, expliquées par des situations géographiques éloignées, ou par des situations géologiques variables.

Pour suivre l'évolution chronologique de la qualité des eaux, les résultats annuels par critère et par puits sont tracés en faisant figurer les valeurs des nombres guides et des concentrations maximales admissibles (CMA) recensées dans les normes de l'Union Européenne et de l'OMS (cf. planche 4).

Même si la qualité des eaux des stockages vérifient quasiunanimement à ces exigences, il reste néanmoins à expliquer toute variation s'écartant significativement des moyennes observées habituellement dans les données de tel ou te] puits.

Pour ce faire il a été fait appel à l'utilisation de méthodes statistiques.

\subsection{L'analyse statistique des données}

Les statistiques utilisées sont de deux types:

- La statistique descriptive, qui a pour but de qualifier les fluctuations liées à l'observation d'un critère, en expliquant si celles-ci sont aléatoires ou inérantes à des cause assignables.
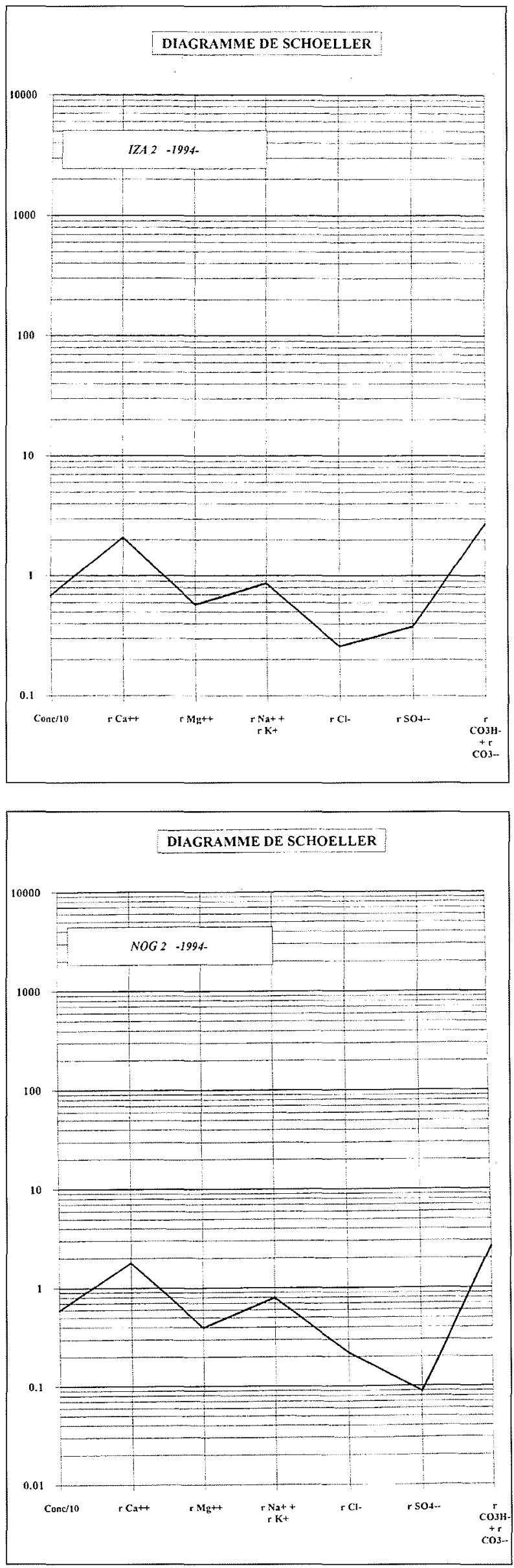


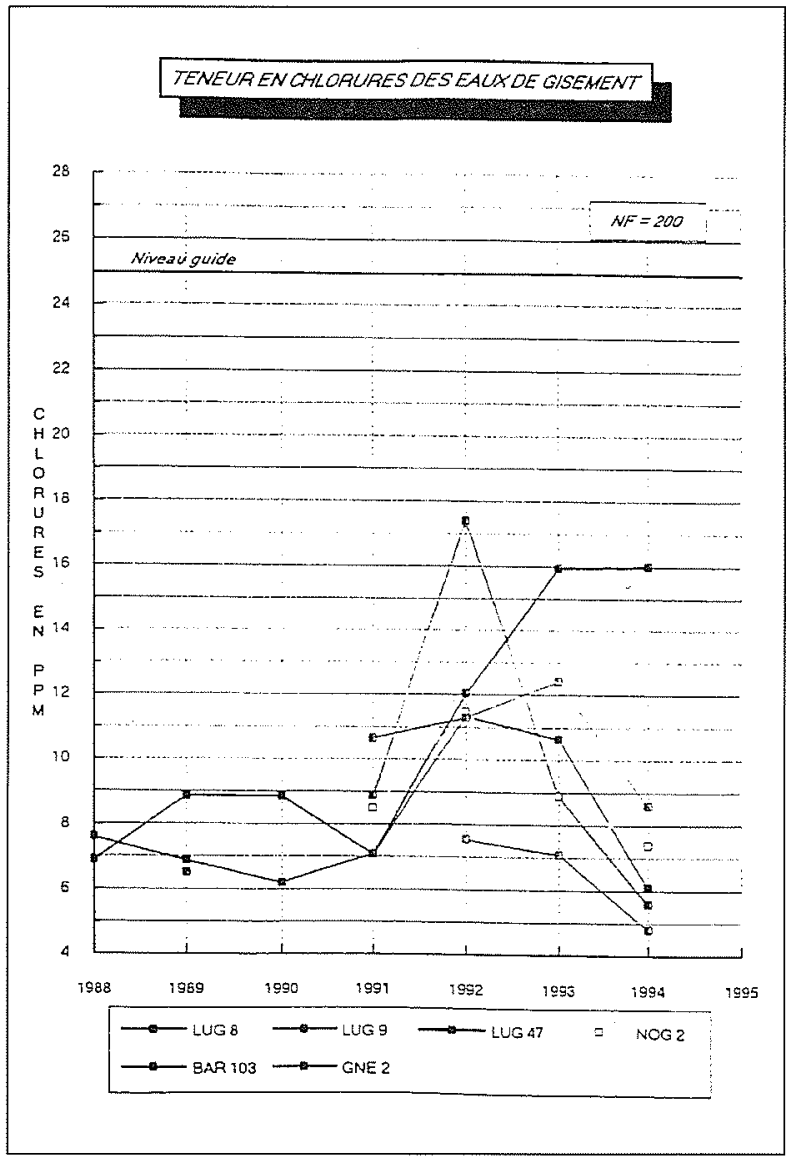

Les principaux outils utilisés sont:

- les tests graphiques ou mathématiques de normalité,

- I'analyse de variance à simple entrée,

- l'étude des corrélations par régression linéaire simple.
- La statistique explicative est destinée à quantifier l'amplitude des phénomènes observés. Les principales techniques utilisées dans ce contexte sont:

- l'analyse de variance,

- l'étude des corrélations.

- l'analyse en composantes principales.

\subsection{Premières conclusions}

Un certain nombre de conclusions ressortent à ce jour. D'une manière générale on peut noter que si on ne constate pas d'évolution significative pour un élément et pour un puits donné, il peut y avoir de différences significatives entre les puits d'un même stockage. Autrement dit si aucune influence tendancielle imputable aux stockages n'a été mise en évidence, il existe un effet-puits, qui reste néanmoins très relatif et ne modifie pas les caractéristiques majeures d'une eat.

Outre cet effet-puits, la plupart des fluctuations observées pour un puits donné entre les relevés d'un même élément d'une année sur l'autre sont imputables aux incertitudes de mesure. Il existe parmi les données acquises au fil du temps des valeurs caractérisées comme aberrantes. Elles s'expliquent par des causes assignables : erreur danalyse, traitement du puits,...

Deux résultats ont appelé une attention particulière

- Le dépassement dans certains puits de la concentration autorisée en fer: les causes du phénomène sont directement attribuables à la corrosion du tubing qui ne se transmet pas de puits à puits, il peut être corrigé.

- Ensuite la corrélation établie sur un puits (Nogaro2) entre les phases d'injection et soutirage et la concentration de sulfates (cf. planche 5).

L’étude spécifique menée sur ce puits a montré qu'il fallait se garder d'assimiler corrélation et causalité : les caractéristiques de ce forage et son exploitation devant être prises en considération. Cette précaution propre à tout traitement statistique n'en a pas moins entraîné un recueil mensuel des donnés sur ce puits qui se poursuivront jusqu'à la mi-1996. afin de bien identifier les fluctuations. A ce jour il semble

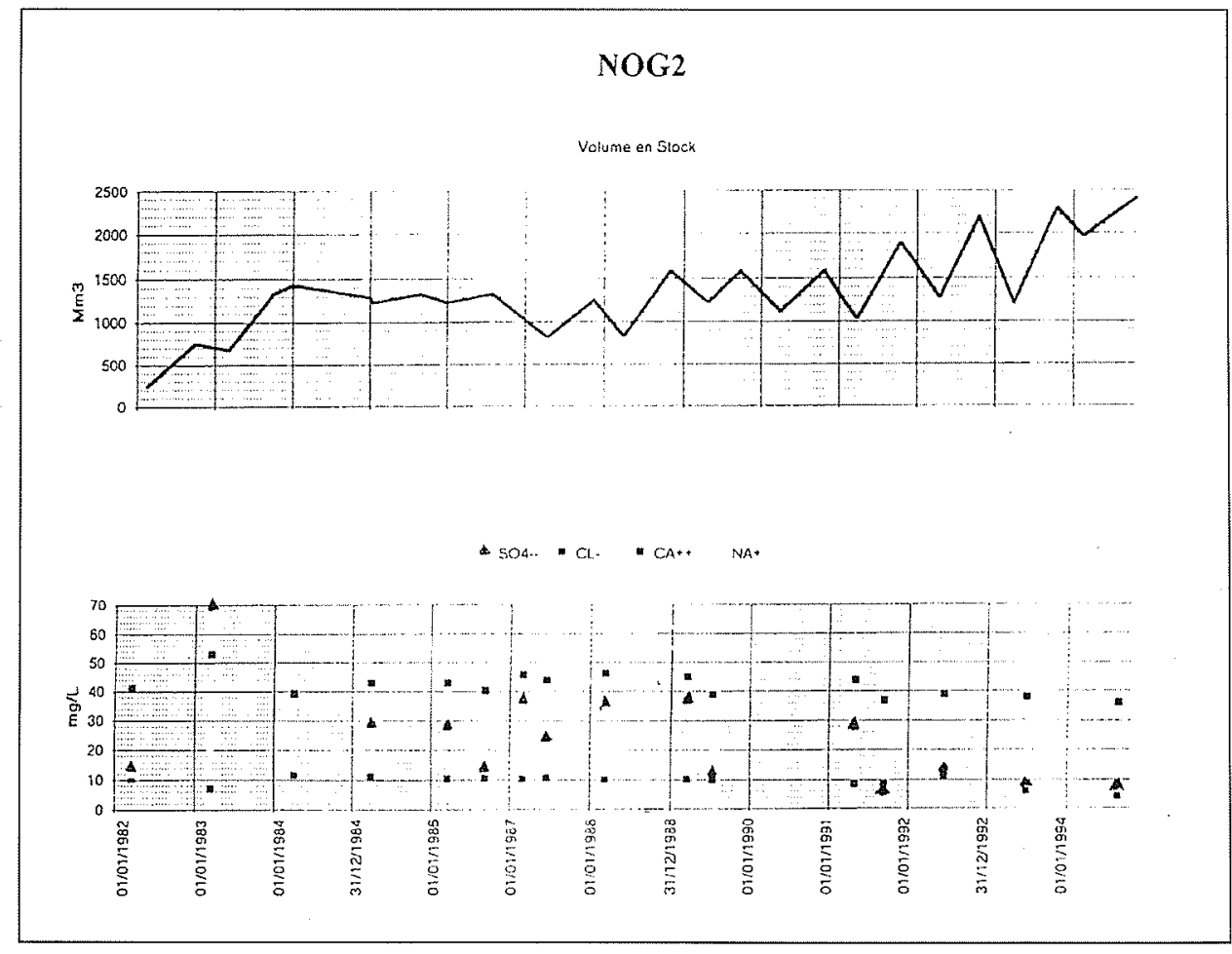


établi que les caractéristiques du puits sont plus explicatives des fluctuations qu'une hypothèse de communication imprévue entre nappes profondes.

Ainsi l'on voit comment le traitement statistique parce qu'il permet de mieux ordonner et rationaliser les données fournies permet au suivi qualitatif de conforter son rôle préventif d'alerte et conduit à améliorer les méthodes d'analyse.

\section{CONTRÔLER ET INFORMER : DES PROCÉDURES PERMANENTES}

Parce que le risque mal connu est générateur d'inquiétudes et que la préoccupation environnementaliste s'accroit, il ne suffit pas de réaliser des travaux de prévention des risques liés à une activité industrielle: il importe tout autant de les faire connaittre. Les réglementations el procédures mises en place par les Pouvoirs Publics peuvent y contribuer. Encore faut-il qu'elles n'aboutissent pas à alourdir à l'excès le coût économique... sans parvenir à réduire les inquiétudes du public.

Il appartient au pétitionnaire d'une autorisation d'exploitation de justifier sa demande et de montrer sa capacité à respecter l'environnement et en assumer les charges.

Il est de la responsabilité des Pouvoirs Publics de donner ou non l'autorisation. C'est à eux d'arbitrer, si nécessaire, entre les besoins du consommateur et les principes de préservation de la nature. Cet arbitrage sera d'autant plus facile que le public se sentira pleinement et clairement informé des enjeux.

Mais au-delà de la première autorisation il convient d'assurer une information permanente comme doit l'être un dispositif de suivi et contrôle apte à prévenir tout dérèglement et ce faisant faciliter la continuité de l'activité industrielle.

\subsection{Le suivi scientifique et technique}

\section{- Le Comité de Suivi de l'Izaute}

Mis en place en 1989. il est chargé du suivi scientifique du stockage. Placé auprès du Ministre de l'Industrie et présidé par un membre du Conseil Général des Mines, il est composé de représentants du Conseil Supérieur d'Hygiène Publique de France, de scientifiques et d'experts cooptés et conseillers de l'Administration. Il propose des recherches, analyse leurs résultats et suggère les dispositions à prendre pour prévenir tout risque vis-à-vis de l'environnement.

\section{- Le Comité des Hydrogéologues}

Plus particulièrement chargé du suivi quantitatif de la ressource en eau et des effets des stockages à cet égard, un Comité des Hydrogéologues a été mis en place au niveau régional en 1993. Sous la direction de la Direction Régionale de l'Industrie et comprenant les experts des collectivités locales concernées, les représentants de l'Agence de l'Eau chargée de la gestion des aquifères par bassin hydrogéologique ainsi que de la Direction Régionale de l'Environnement, il vérifie les résultats et propose des aménagements du suivi quantitatif.

Au moins une fois par an et autant que de besoin Elf présente à ces instances les données fournies par le réseau de suivi et propose les interprétations, analyses ou interrogations qu'elles provoquent.

\section{- Le Comité Départemental d'Hygiène}

Les Comités Départementaux d'Hygiène sont, sous la présidence du Préfet des départements concernés, informés chaque année des conclusions des travaux du Comité de Suivi et des Comités des Hydrogéologues. Composés de représentants des élus et des associations locales, ils devraient être le reflet des préoccupations du grand public.

\subsection{Le suivi administratif}

En période d'accroissement du-volume stocké, l'exploitant des stockages doit rendre compte chaque année gazière auprès des administrations locales du bilan de l'année gazière passée et du programme d’injection et soutirage prévu pour l'année gazière à venir.

En accordant leur autorisation au programme prévu, les représentants locaux du Ministère de l'industrie fixent le volume et les pressions autorisés. Ils vérifient que le dispositif de suivi du stockage est opérationnel comme ils s'assurent de la conformité et la sécurité des installations de surface et de la qualité du gaz injecté ou soutiré pour la distribution.

Ce faisant, ils arrêtent les dispositions dont le non respect entraînerait des pénalités pour l'exploitant et assurent auprès du public le souci d'une exploitation conforme aux règles de sécurité.

Cette construction institutionnelle est récente. Elle mérite sûrement d'être précisée et améliorée autour de trois préoccupations :

- éviter les double emplois générateurs de surcoûts, voire de conflits entre instances,

- intégrer plus efficacement les résultats du suivi continu et les procédures d'autorisation,

- assurer que la transparence n'est pas confinée à quelques spécialistes mais que l'information parvient à tous ceux qui peuvent être concernés.

\section{CONCLUSION}

Au travers de notre expérience, nous avons vu progressivement s'affirmer la nécessité de répondre simultanément à deux préoccupations:

- assurer l'approvisionnement en gaz naturel d'un nombre croissant d'utilisateurs nécessitant une modulation saisonnière,

- préserver la qualité et les quantités disponibles d'une ressource essentielle destinée à la consommation humaine.

Longtemps on a pu croire que le probleme ne se posait pas. Puis certains ont considéré que ces deux affirmations étaient antinomiques. Les raisons de cette contradiction ont varié dans le temps, le poids des arguments hydrologiques remplaçant peu à peu les arguments liés aux risques de contamination de l'eau par le gaz, qui avaient eux-mêmes pris le pas sur les craintes provoquées par les installations de surface du stockage. II s'agit moins aujourd'hui de hiérarchiser le poids des différents risques que, en ayant mesuré la portée réelle, les couvrir tous au travers d'une prévention dynamique.

La mise en place d'un dispositif de suivi dont la fiabilité scientifique et technique est reconnue et dont la transparence des résultats est assurée permet et permettra de concilier ce double besoin de confort et de sécurité qu'expriment les usagers. 